\title{
Pseudomonas conjunctival ulcer and secondary orbital cellulitis in a patient with AIDS
}

\author{
Juan Cano-Parra, Enrique España, Miguel Esteban, Manuel Díaz-Llopis, Jose L Menezo
}

Bacterial infections of the anterior segment of the eye are not a common feature of AIDS but once established these infections may be particularly severe and more likely to cause ulcer and perforation. ${ }^{1}$ Pseudomonas aeruginosa has been cultured in keratitis, corneal ulcers, and scleritis in HIV infected patients ${ }^{2}$; nevertheless, nobody has previously reported Pseudomonas conjunctival ulcers in HIV patients.

\section{Case report}

A 26-year-old man with a history of intravenous drug misuse was diagnosed as having AIDS a year before presentation after one episode of Pneumocystis carinii pneumonia. He also had cachexia, oral candidiasis with secondary anaemia, and neutropenia due to treatment with zidovudine. He presented himself to the ophthalmologist on 29 June 1992, with a 2 day history of severe pain and a greenish discharge from his right eye. No history of trauma, contact lens, or previous ocular problems was elicited. Best corrected visual acuity was 20/25 in the right eye and 20/20 in the left eye. The patient exhibited signs of orbital cellulitis in the right eye, including periocular erythema, ptosis, proptosis of $3 \mathrm{~mm}$, eyelid oedema, tenderness, total ophthalmoplegia, and chemosis. Slit-lamp examination disclosed purulent conjunctivitis with a conjunctival and Tenon's capsule ulcer in the nasal side of the right eye that measured $5 \times 8 \mathrm{~mm}$ (Fig 1). The corneal examination did not show any abnormal findings. The left eye appeared normal. Computed tomography of the orbits disclosed an enhancing mass lesion in the anteronasal region of the right orbit without sinus involvement or bony erosion, more consistent with an orbital cellulitis than an orbital abscess (Fig 2). Laboratory studies revealed a leucocyte count of $1 \cdot 1 \times 10^{9} / 1$ (58\% polymorphonuclear leucocytes). The absolute CD4+ lymphocyte count was $155 \times 10^{6} / 1$. Empirical treatment before conjunctival culture results was as follows: fortified tobramycin $15 \mathrm{mg} / \mathrm{ml}$ and cefazolin sodium $50 \mathrm{mg} / \mathrm{ml}$ one drop of each in

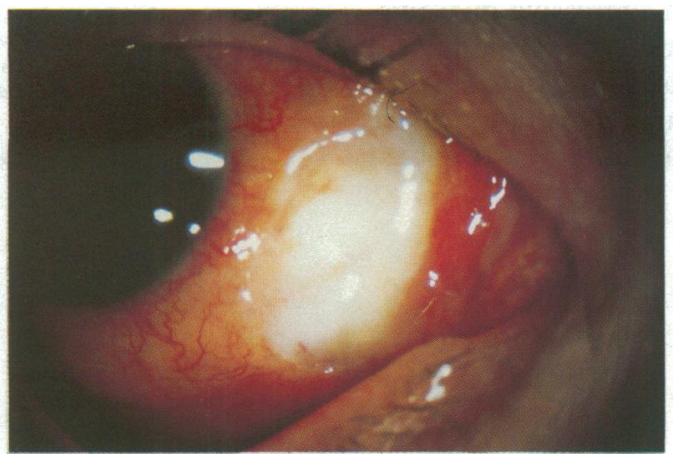

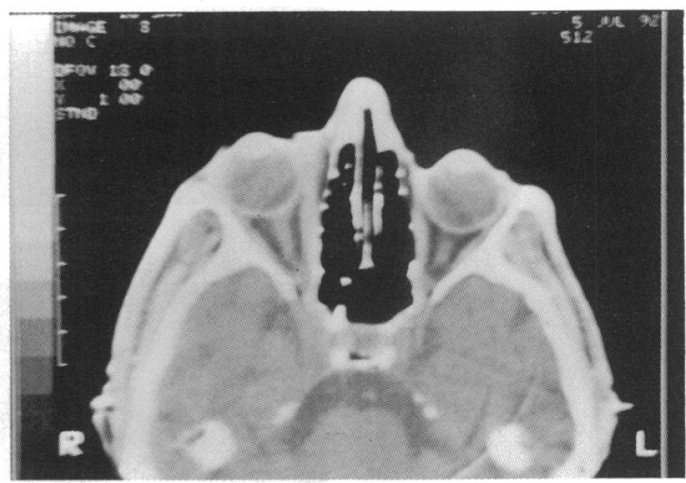

Figure 2 Computed tomogram showing the proptosis and the hyperdense enhancing mass lesion in the anteronasal region of the right orbit without sinus involvement or bony erosion.

the right eye every hour around the clock and intravenous ciprofloxacin $200 \mathrm{mg}$ every 12 hours. In 24 hours, cultures of the ulcer grew Pseudomonas aeruginosa sensitive to tobramycin, ceftazidime, and ciprofloxacin, so we replaced cefazolin with ceftazidime. In 48 hours, the discharge, the proptosis, and the ductional restriction diminished. Eight days later the conjunctival culture was negative and the ophthalmic examination was normal.

\section{Comment}

Pseudomonas corneal ulcers have previously been reported in neutropenic patients infected with HIV' but, to our knowledge, this is the first case of Pseudomonas conjunctival ulcer complicated with an orbital cellulitis. Other acute orbital involvements in AIDS such as pseudotumour, ${ }^{3}$ aspergillosis, ${ }^{4}$ and Pneumocystis carinii of the orbit $^{5}$ were considered in the differential diagnosis, but our diagnosis was based on conjunctival cultures, clinical and computed tomography findings, and patient's prompt and complete response to a specific antibiotic therapy. The importance of neutrophils in the clearing of this invasive bacterial infection is well known. Previous studies showed that in experimental infection of neutropenic rodents and hamsters, the administration of recombinant granulocyte colony stimulating factor ( $\mathrm{rG}-\mathrm{CSF}$ ) increased survival rates. $\mathrm{rG}-\mathrm{CSF} \quad 0.4 \mathrm{mg} / \mathrm{kg}$ administered at the same time as, and for 2 consecutive days following, infection with Pseudomonas aeruginosa resulted in $46 \%$ survival 1 week after infection, compared with $6 \%$ survival in control mice. Leucocyte numbers in both control and rG-CSF treated mice increased up to 15 hours after infection, although levels in rG-CSF treated mice were always higher than those in controls. ${ }^{6}$ Furthermore, Pseudomonas produces several virulent factors: cytotoxin, a 25000 molecular weight protein originally
Figure 1 Conjunctival and Tenon's capsule ulcer in the nasal side, that measured

$5 \times 8 \mathrm{~mm}$.

\author{
La Fe Hospital, \\ J Cano-Parra \\ E España \\ School of Medicine \\ M Díaz-Llopis
}


termed leucocidin because of its cytopathic effects on polymorphonuclear leucocytes, and the production of elastase and an alkaline protease, which inhibit neutrophil chemotaxis in vitro. These characteristic factors of Pseudomonas along with the neutropenia may explain this unusual case. We did not use rG-CSF in this patient because of his prompt response to antibiotic therapy. However, we consider that the use of rG-CSF in combination with antibiotic therapy in severe neutropenic patients with life threatening infection is mandatory because it minimises the risk of infection and decreases the mortality rates. We therefore conclude that an early diagnosis is necessary, as well as aggressive treatment, because a Pseudomonas infection in a neutropenic patient manifests a truly fulminating progression.
1 Maguem E, Saltz JJ, Nesburn AB. Pseudomonas corneal ulcer associated with rigid, gas-permeable, daily-wear lenses in a patient infected with human immunodeficiency virus. Am F Ophthalmol 1992; 113: 336-7.

2 Nanda M, Pflugfelder SC, Holland S. Fulminant pseudomonal keratitis and scleritis in human immunodeficiency virusinfected patients. Arch Ophthalmol 1991; 109: 503-5.

3 Benson WH, Linberg JV, Weinstein GW. Orbital pseudotumo in a patient with AIDS. Am $\mathcal{F}$ Ophthalmol 1988; 105: 697-8.

Vitale AT, Spaide RF, Warren FA, Moussouris HF, D'Amico RA. Orbital aspergillosis in an immunocompromised host. Am f Ophthalmol 1992; 113: 725-6.

5 Friedberg DN, Warren FA, Lee MH, Vallejo C. Pneumocystis carinii of the orbit. Am F Ophthalmol 1992;113: 595-6.

6 Yasuda H, Ajiki T, Shimozato T, Kasahara M, Kawada H Iwater $M$, et al. Therapeutic efficacy of granulocyte colonystimulating factor alone and in combination with antibiotics against Pseudomonas aeruginosa infections in mice. Infec tion and Immunity 1990; 58: 2502-9.

7 Pollack M. Pseudomonas aeruginosa. In: Mandell GL, Douglas RG, Bennet JE, eds. Principles and practice of infectious diseases. 3rd ed. Edinburgh: Churchill Livingstone, 1990 1673-91.

\title{
Ocular melanocytosis and cavernous haemangioma of the optic disc
}

\author{
Leonidas Zografos, Michel Gonvers
}

Jules Gonin Eye Hospital, University of Lausanne, Switzerland L Zografos

$M$ Gonvers

Correspondence to:

Dr Leonidas Zografos, Jules Gonin Eye Hospital, Avenue de France 15, CH-1004 Lausanne, Switzerland.

Accepted for publication 17 August 1993
Figure 1 Right eye of the patient demonstrating a diffuse perilimbal episcleral pigmentation.
Cavernous haemangioma of the optic disc is a rare vascular tumour that may occasionally produce a vitreous haemorrhage. ${ }^{12}$ Ocular melanocytosis, on the other hand, is a more common condition but one that is associated with an increased incidence of uveal melanoma..$^{3-5} \mathrm{We}$ encountered an unusual case in which ocular melanocytosis was associated with a cavernous haemangioma of the optic disc that had been masked behind a dense vitreous haemorrhage.

\section{Case report}

A 38-year-old man was referred with a diagnosis of vitreous haemorrhage of the right eye due to a tumour of the optic disc. Over the past 7 years,

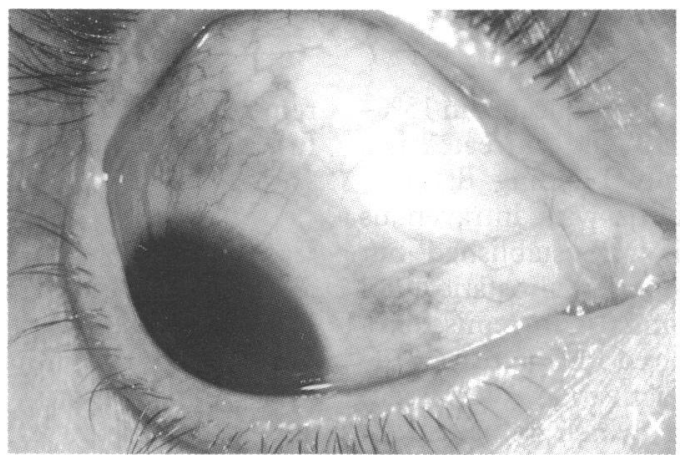

he had been seen on several occasions with vitreous haemorrhages, each of which had resorbed spontaneously.

When first examined by us, visual acuity in the right eye was finger counting. The diagnosis of ocular melanocytosis was based upon the presence of several dark scleral flecks (Fig 1) and iris hyperchromia. Visualisation of the fundus was obscured by a vitreous haemorrhage, and B scan ultrasonography disclosed a $4.5 \mathrm{~mm}$ thick mass covering the optic disc (Fig 2). The anterior two thirds of the mass were moderately reflective,

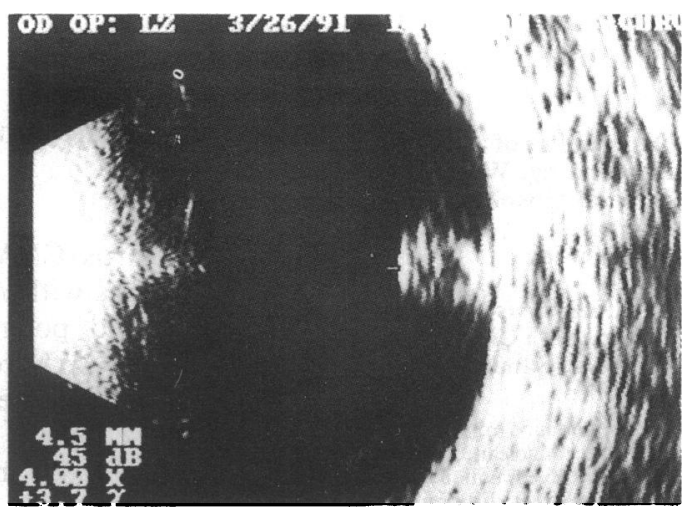

Figure 2 B scan ultrasonography. A $4.5 \mathrm{~mm}$ thick tumour mass covers the optic nerve. Internal reflectivity of the base of the tumour is high. 